\title{
Spurious electroencephalographic activity due to pulsation artifact in the depth of anesthesia monitor
}

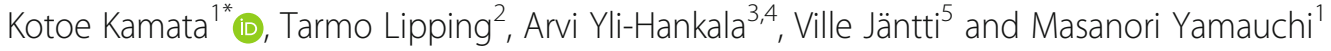

\begin{abstract}
Background: The depth of anesthesia (DOA) is estimated based on the anesthesia-induced electroencephalogram (EEG) changes. However, the surgical environment, as well as the patient him/herself, generates electrical interferences that cause EEG waveform distortion.

Case presentation: A 52-year-old patient required general anesthesia due to the right femur necrotizing fasciitis. He had no history of epilepsy or head injury. His cardiovascular status was stable without arrhythmia under propofol and remifentanil anesthesia. The DOA was evaluated with Root ${ }^{\oplus}$ with SedLine ${ }^{\oplus}$ Brain Function Monitoring (Masimo Inc, Irvine, CA). The EEG showed a rhythmic, heart rate time-locked pulsation artifact, which diminished after electrode repositioning. Offline analysis revealed that the pulse wave-like interference in EEG was observed at the heart rate frequency.

Conclusions: We experienced an anesthesia case that involves a pulsation artifact generated by the superficial temporal artery contaminating the EEG signal. Numerous clinical conditions, including pulsation artifact, disturb anesthesia EEG.
\end{abstract}

Keywords: Artifact, Depth of anesthesia, Electroencephalogram, Intraoperative, Monitoring, Pulse wave

\section{Background}

Several kinds of monitors have been developed to measure the depth of anesthesia (DOA). These monitors calculate indices from a large electroencephalogram (EEG) database using proprietary software [1-3]. Previous reports show inaccurate EEG-derived indices caused by waveform distortion. High facial muscle activities and electrical noise are the leading causes of artifacts on anesthesia EEG [4]. Here we experienced an anesthesia case that involves a pulsation artifact generated by the superficial temporal artery (STA) contaminating EEG signal.

\footnotetext{
* Correspondence: kotoe.kamata.c3@tohoku.ac.jp

'Department of Anesthesiology and Perioperative Medicine, Tohoku University School of Medicine, 2-1 Seiryo-machi, Aoba-ku, Sendai-shi, Miyagi 980-8575, Japan

Full list of author information is available at the end of the article
}

\section{Case presentation}

The study was approved by the Ethics Committee of Tohoku University School of Medicine (No. 21334). The written informed consent was obtained from the patient for publication of this case report and any accompanying images. A 52-year-old male $(180 \mathrm{~cm}, 84.2 \mathrm{~kg})$ suffered from septic shock due to right femur necrotizing fasciitis. He had no history of epilepsy or head injury. Preoperative brain computed tomography and chest X-ray revealed no abnormalities. Electrocardiogram (ECG) showed a normal sinus rhythm. Five days after the initial operation of the right below-knee amputation, debridement was planned for local infection control. He was classified as American Society of Anesthesiologists (ASA) Physical Status Class 3E.

Under intravenous sedation with dexmedetomidine $\left(12 \mu \mathrm{g} \mathrm{h}^{-1}\right)$ and fentanyl $\left(100 \mu \mathrm{g} \mathrm{h}{ }^{-1}\right)$, the patient successfully adapted to a mechanical ventilator with

\section{Springer Open}

(c) The Author(s). 2021 Open Access This article is licensed under a Creative Commons Attribution 4.0 International License, which permits use, sharing, adaptation, distribution and reproduction in any medium or format, as long as you give appropriate credit to the original author(s) and the source, provide a link to the Creative Commons licence, and indicate if changes were made. The images or other third party material in this article are included in the article's Creative Commons licence, unless indicated otherwise in a credit line to the material. If material is not included in the article's Creative Commons licence and your intended use is not permitted by statutory regulation or exceeds the permitted use, you will need to obtain permission directly from the copyright holder. To view a copy of this licence, visit http://creativecommons.org/licenses/by/4.0/. 
endotracheal intubation. Besides standard ASA monitoring, invasive blood pressure was continuously recorded by an automated anesthesia recording system (PRM-7000; Nihon Kohden Co., Tokyo, Japan). The DOA was evaluated with $\operatorname{Root}^{\circ}$ with SedLine ${ }^{\oplus}$ Brain Function Monitoring Version 1.8.1.4i (Masimo Inc, Irvine, CA), as a standalone monitor. Surgical anesthesia was induced with additional propofol $\left(500 \mathrm{mg} \mathrm{h}^{-1}\right)$, remifentanil $\left(1 \mathrm{mg} \mathrm{h}^{-1}\right)$, and rocuronium $(50 \mathrm{mg})$. Continuous infusion of phenylephrine (1 $\mathrm{mg} \mathrm{h}^{-1}$ ) controlled the intraoperative hemodynamics while maintaining his mean arterial pressure within $25 \%$ of the baseline value (i.e., hypotension-related EEG abnormalities were minimized). His heart rate was stable at around $64 \mathrm{bpm}$ without arrhythmia. The EEG showed a rhythmic, heart rate time-locked pulsation artifact over one of four traces, L2, when there was a momentary pause in the electrocautery interferences (Fig. 1a). Arterial pulsation was palpable beneath the L2 electrode. The electrode impedance and cable connection were checked, and the pulse wave-like artifact diminished after repositioning the L2 electrode. Thereafter, all four traces showed the same EEG pattern as the burst-suppression (Fig. 1b). The PSi, a DOA index calculated by SedLine ${ }^{\circ}$ Brain Function Monitoring, did not remarkably change. No electrolyte imbalance or hypoglycemia $\left(195 \mathrm{mg} \mathrm{dL}^{-1}\right.$ ) was observed. Arterial blood gas analysis showed an acceptable level of oxygenation. Four days after the second operation, a full recovery, without neurological deficits, was confirmed following extubation.

Numerical values recorded by PRM-7000 and EEG data were exported from SedLine ${ }^{\bullet}$ using the European Data Format and analyzed offline using MATLAB (Mathworks, Natick, MA, USA). Details of the four EEG channels, ECG, and plethysmography before and after the electrode replacement are shown for two 10-s samples (left and right images) in Fig. 1c. To show time locking between the slow-wave EEG and ECG, the EEG signal was first filtered using a linear-phase low-pass filter (cut-off frequency $1.5 \mathrm{~Hz}$ ), then the Hilbert transform was applied to obtain the analytic signal and the instantaneous frequency was expressed as the digital derivative of the phase of the analytic signal. Finally, the instantaneous frequency curve was smoothed using a moving average over a 4 -s window. The instantaneous frequency of the low-frequency component of L2 coincided with the heart rate due to the pulsation artifact and was separated after electrode replacement (Fig. 1d).

\section{Discussion}

This case shows that arterial pulsation can cause an artifact, resulting in anesthesia EEG misinterpretation. The single EEG trace with a regularly occurring slowwave synchronously with the patient's pulse was noticeable. Usually, signal contamination in EEG-derived anesthesia monitors is automatically filtered using proprietary software. The current case shows that removing such artifact is impossible due to automatic filtering from the EEG, which is used in the specific processedEEG monitor, SedLine ${ }^{\circ}$ Brain Function Monitoring. Moreover, the fact that the PSi value did not change after electrode replacement suggests the PSi calculation algorithm's robustness. Common artifactual noise, such as frontal muscle contraction or electrocautery, affects several EEG leads because they are not generated within a limited area $[5,6]$, whereas a pulse wave derivative can be recorded from a single electrode if placed just over the artery. Other possible etiologies of the focal electrical activities that occur in frontal, pathological lesions or motor unit potential have also been suggested. A misinterpretation of EEG-derived index caused by epileptiform activity has been recorded under sevoflurane anesthesia [7]. A cerebrospinal fluid pulsation of frontal pseudomeningocele could also cause an artifact similar to an ictal EEG discharge [8]. If a single motor unit potential is regularly firing under the EEG electrode, spiky electromyograph artifacts are visible overlying EEG [9]. Concurrent contraction of several motor units, including larger motor units, may no longer show a periodic wave [10].

The case-patient showed no seizure-like phenomena and epileptiform activity was not seen in other leads. The pulse wave-like interference in EEG was observed at the heart rate frequency (i.e., 64 cycles $\mathrm{min}^{-1}$ or $1.1 \mathrm{~Hz}$, approximately) and occurred only in the L2 electrode. The SedLine ${ }^{\circ}$ is a distinct DOA monitor with four active EEG leads to collect and process signals from the bilateral frontal lobe. The manufacturer's manual recommends that the L2 and R2 electrodes be applied to the hairless region just above the left and right temple, respectively. However, STA is one of the two terminal branches of the external carotid artery, and the level and size of these terminal branches differ. Still, the anterior branch, which commonly runs anterosuperiorly, supplying muscles, pericranium, and skin of the lateral frontal area, could be beneath the L2 or R2 electrode. Checking the STA frontal branch pulsation before the electrodes are applied may diminish excessive noise contamination.

The pulsatile artifact was easily detected during visual diagnosis in this case for two reasons: (1) The irregularly contaminated artifacts are hardly distinguishable in patients with arrhythmia. However, the case-patient did not have an arrhythmia. (2) The background EEG waveforms can create prominent artifacts because of burstsuppression or isoelectric EEG pattern. Figure 1d indicates a possible way to detect the pulsatile artifact by comparing the reciprocal of the averaged instantaneous frequency of the low-frequency component of the EEG with the heart rate. 
a

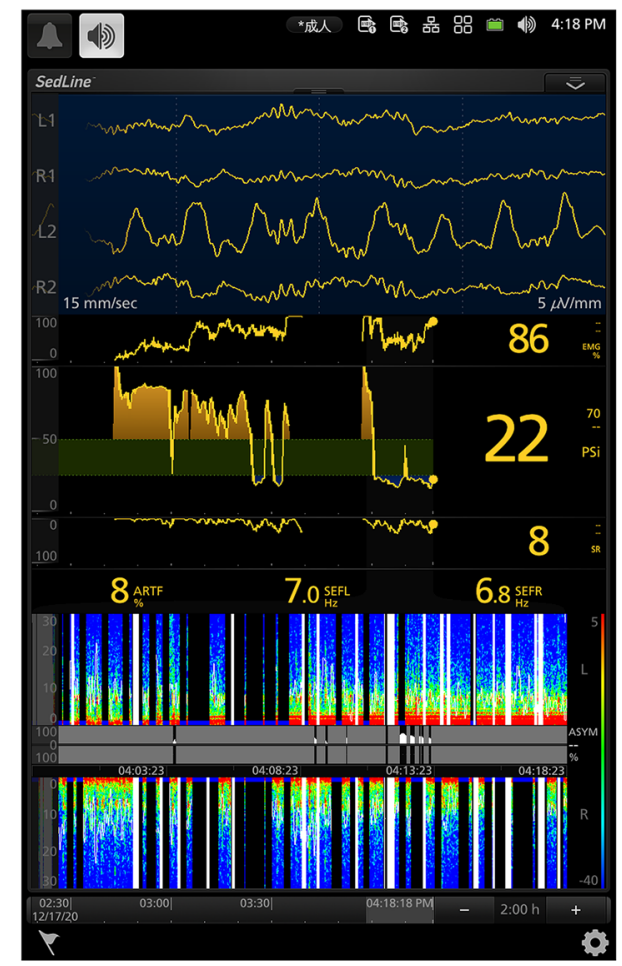

c

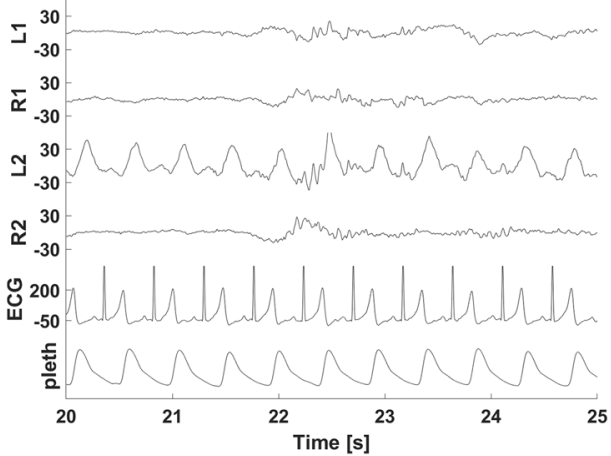

b

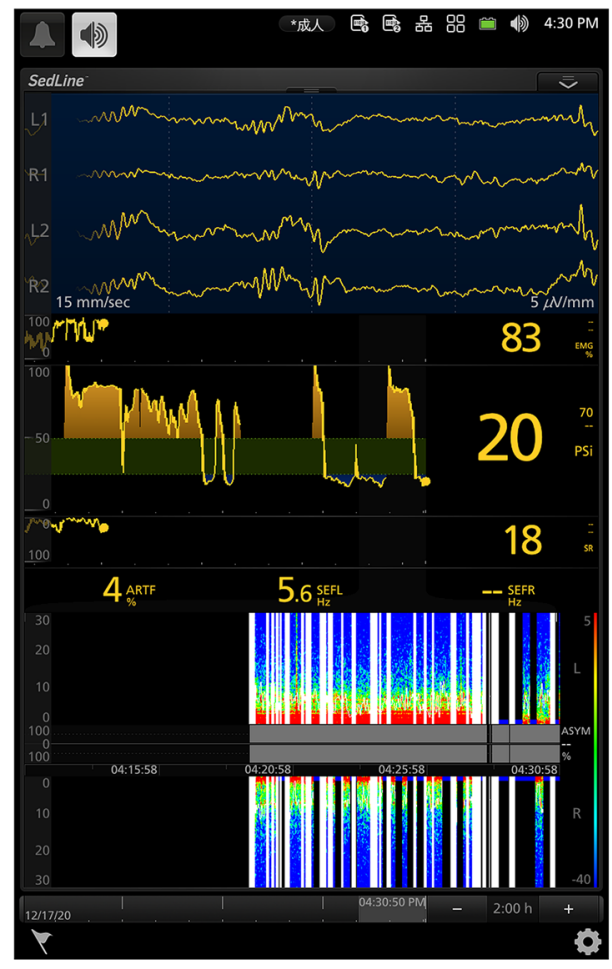

$\tau_{-30}^{30}$

$\bar{x}_{-30}^{30}$

$\Im_{-30}^{30}$

ฯ 30 -

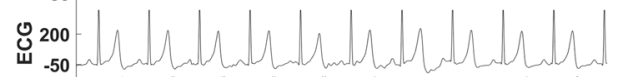

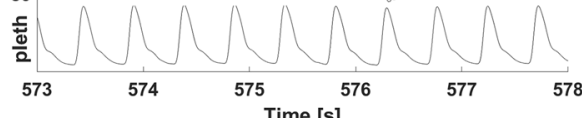

d

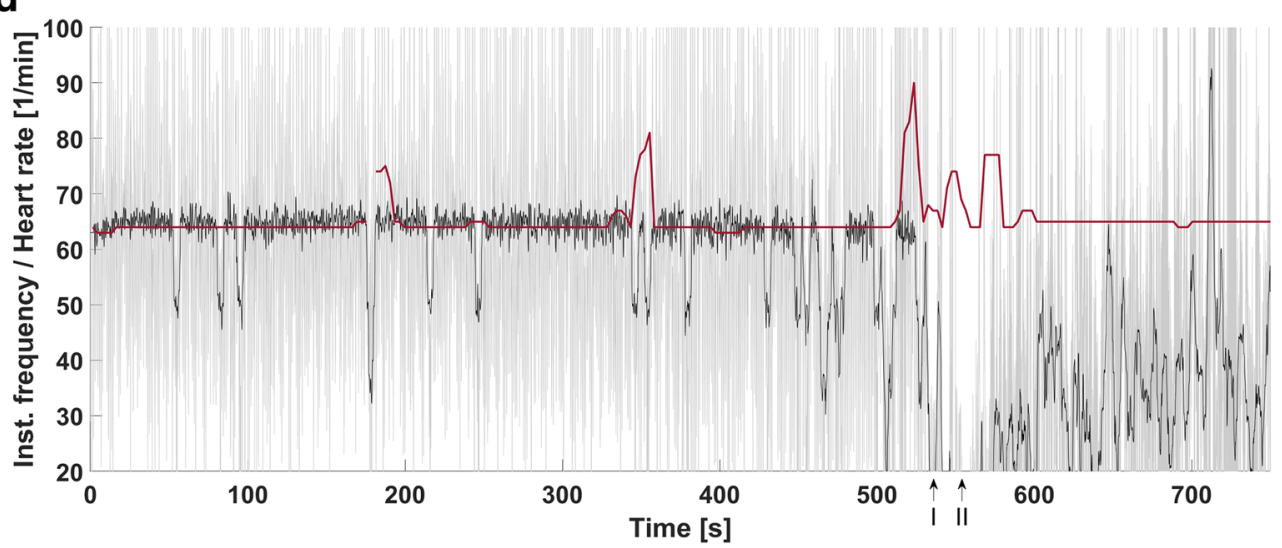

Fig. 1 (See legend on next page.) 
(See figure on previous page.)

Fig. 1 Normalization of electroencephalography after L2 electrode repositioning. a A snapshot of the Root $^{\oplus}$ with SedLine ${ }^{\oplus}$ Brain Function Monitoring (Masimo Inc, Irvine, CA) is presented. A rhythmic, heart rate time-locked pulsation artifact is overlying L2. b Following L2 electrode repositioning, a pulsation artifact has completely disappeared. All four electroencephalogram (EEG) traces show a burst-suppression pattern. $\mathbf{c}$ Two 10-s samples (left and right) of four EEG channels (L1, R1, L2, and R2), electrocardiogram (ECG), and plethysmography (pleth) show how the waveform of $L 2$ changes after electrode replacement. The synchronization between ECG/pleth versus EEG is not perfect due to separate standalone monitoring devices, but it can be seen that the pace of the low fluctuation in L2 EEG is similar to that of the heart rate and pleth. The units of EEG and ECG signals are microvolts. $\mathbf{d}$ Heart rate (red) and instantaneous frequency of the low-frequency component of L2. Both sampleby-sample estimate and smoothed version over a 4-s window are shown in black color. The time axis values correspond to those in $\mathbf{c}$ above. The Roman numerals I and II indicate the timepoints of making the start of the electrode replacement (I) and recovery of the EEG signal (II), respectively

\section{Conclusions}

Numerous clinical conditions, including pulsation artifact, have direct effects on EEG waveform. Sole monitoring of numerical values from DOA monitors may be unreliable; therefore, raw changes in EEG signal should be evaluated to accurately measure the DOA.

\section{Abbreviations}

ASA: American Society of Anesthesiologists; DOA: Depth of anesthesia; ECG: Electrocardiogram; EEG: Electroencephalogram; STA: Superficial temporal artery

\section{Acknowledgements}

Not applicable.

\section{Authors' contributions}

KK coordinated the patient care, prepared the manuscript, and obtained informed consent. TL analyzed and interpreted the patient data regarding anesthesia EEG interferences. AY-H interpreted the patient data and helped to draft the manuscript. $V J$ interpreted the patient data and was a major contributor in writing the manuscript. MY helped to draft the manuscript. All authors read and approved the final manuscript.

\section{Authors' information}

Not applicable.

\section{Funding}

The present report was supported solely by the hospital and/or departmental sources.

\section{Availability of data and materials}

The datasets used and analyzed during the current study are available from the corresponding author on reasonable request.

\section{Declarations}

\section{Ethics approval and consent to participate}

The study was approved by the Ethics Committee of Tohoku University School of Medicine (No. 21334).

\section{Consent for publication}

Informed consent was obtained from the patient for publication of this case report and any accompanying images. A copy of the written consent is available for review from the Editor-in-Chief of this journal.

\section{Competing interests}

The authors declare that they have no competing interests.

\section{Author details}

${ }^{1}$ Department of Anesthesiology and Perioperative Medicine, Tohoku University School of Medicine, 2-1 Seiryo-machi, Aoba-ku, Sendai-shi, Miyagi 980-8575, Japan. ${ }^{2}$ Faculty of Information Technology and Communication, Tampere University, Pohjoisranta 11, 28100 Pori, Finland. ${ }^{3}$ Department of Anesthesia, Tampere University Hospital, Elämänaukio 2, 33520 Tampere, Finland. ${ }^{4}$ Faculty of Medicine and Health Technology, Tampere University,
Kalevantie 4, 33100 Tampere, Finland. ${ }^{5}$ Department of Clinical Neurophysiology, Seinäjoki Central Hospital, Hanneksenrinne 7, 60220 Seinäjoki, Finland.

Received: 30 March 2021 Revised: 7 April 2021

Accepted: 8 April 2021 Published online: 17 April 2021

\section{References}

1. Johansen JW, Sebel PS. Development and clinical application of electroencephalographic bispectrum monitoring. Anesthesiology. 2000; 93(5):1336-44. https://doi.org/10.1097/00000542-200011000-00029.

2. Prichep LS, Gugino LD, John ER, Chabot RJ, Howard B, Merkin H, et al. The Patient State Index as an indicator of the level of hypnosis under general anaesthesia. Br J Anaesth. 2004;92(3):393-9. https://doi.org/10.1093/bja/aeh082.

3. Viertio-Oja H, Maja V, Sarkela M, Talja P, Tenkanen N, Tolvanen-Laakso H, et al. Description of the Entropy algorithm as applied in the Datex-Ohmeda S/5 Entropy Module. Acta Anaesthesiol Scand. 2004;48(2):154-61. https://doi. org/10.1111/j.0001-5172.2004.00322.x.

4. Aho AJ, Lyytikainen LP, Yli-Hankala A, Kamata K, Jantti V. Explaining Entropy responses after a noxious stimulus, with or without neuromuscular blocking agents, by means of the raw electroencephalographic and electromyographic characteristics. Br J Anaesth. 2011;106(1):69-76. https:// doi.org/10.1093/bja/aeq300.

5. Chan MT, Ho SS, Gin T. Performance of the bispectral index during electrocautery. J Neurosurg Anesthesiol. 2012;24(1):9-13. https://doi.org/10.1 097/ANA.0b013e31823058bf.

6. Cristakos CNL, S. Lumped and population stochastic models of skeletal muscle: implications and predictions. Biol Cybern. 1980;36(2):73-85. https:// doi.org/10.1007/BF00361076.

7. Sarkela MO, Ermes MJ, van Gils MJ, Yli-Hankala AM, Jantti VH, Vakkuri AP. Quantification of epileptiform electroencephalographic activity during sevoflurane mask induction. Anesthesiology. 2007;107(6):928-38. https://doi. org/10.1097/01.anes.0000291444.68894.ee.

8. Wallace A, Shin C, Wirrell EC, Burkholder DB. False ictal-appearing EEG from a frontal sinus pseudomeningocele. Neurology. 2016;87(24):2600-1. https://doi.org/10.1212 WNL.0000000000003437.

9. Kamata K, Aho AJ, Hagihira S, Yli-Hankala A, Jantti V. Frequency band of EMG in anaesthesia monitoring. Br J Anaesth. 2011;107(5):822-3. https://doi. org/10.1093/bja/aer311.

10. Goncharova II, McFarland DJ, Vaughan TM, Wolpaw JR. EMG contamination of EEG: spectral and topographical characteristics. Clin Neurophysiol. 2003; 114(9):1580-93. https://doi.org/10.1016/S1388-2457(03)00093-2.

\section{Publisher's Note}

Springer Nature remains neutral with regard to jurisdictional claims in published maps and institutional affiliations. 